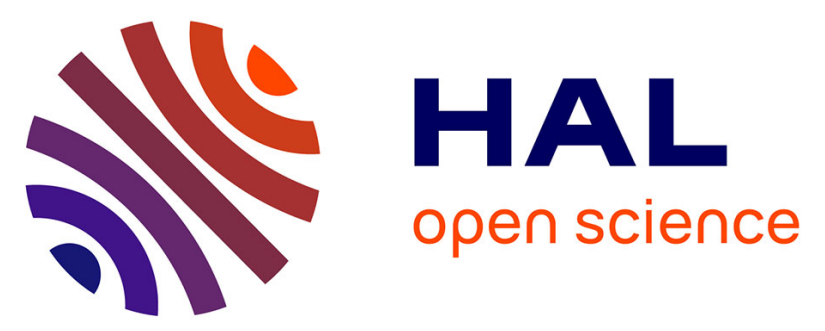

\title{
Towards a Spatiotemporal Ontology-Based on Mereotopological Theory in Assembly-Oriented Design
}

Elise Gruhier, Frédéric Demoly, Olivier Dutartre, Said Abboudi, Samuel

Gomes

\section{- To cite this version:}

Elise Gruhier, Frédéric Demoly, Olivier Dutartre, Said Abboudi, Samuel Gomes. Towards a Spatiotemporal Ontology-Based on Mereotopological Theory in Assembly-Oriented Design. IFIP International Conference on Advances in Production Management Systems (APMS), Sep 2014, Ajaccio, France. pp.225-232, 10.1007/978-3-662-44739-0_28 . hal-01388250

\section{HAL Id: hal-01388250 \\ https://hal.inria.fr/hal-01388250}

Submitted on 26 Oct 2016

HAL is a multi-disciplinary open access archive for the deposit and dissemination of scientific research documents, whether they are published or not. The documents may come from teaching and research institutions in France or abroad, or from public or private research centers.
L'archive ouverte pluridisciplinaire HAL, est destinée au dépôt et à la diffusion de documents scientifiques de niveau recherche, publiés ou non, émanant des établissements d'enseignement et de recherche français ou étrangers, des laboratoires publics ou privés.

\section{(c)(1)}

Distributed under a Creative Commons Attribution| 4.0 International License 


\title{
Towards a Spatiotemporal Ontology-based on Mereotopological Theory in Assembly-Oriented Design
}

\author{
Elise Gruhier, Frédéric Demoly, Olivier Dutartre, Said Abboudi, and Samuel \\ Gomes \\ IRTES-M3M, \\ UTBM 90010 Belfort Cedex, France \\ \{elise.gruhier, frederic.demoly, olivier.dutartre, said.abboudi, samuel. \\ gomes\}@utbm.fr
}

\begin{abstract}
This paper presents a novel spatiotemporal ontology based on a mereotopological theory in the context of assembly-oriented design, which integrates assembly sequence planning in the early product design stages. Based on a brief literature review on ontology and existing spatiotemporal ontological models, the authors propose to go beyond by defining their own formal ontology in the domain of assembly-oriented design. The proposed ontology provides formal description of productprocess information and information consistency checking through the product lifecycle. Here, the ontology covers the spatial, temporal and spatiotemporal dimensions. The ontology uses OWL language and is implemented in Protégé. The main objective is to provide a product design description by proactively considering its assembly sequence as early as possible in the product development so as to ensure information and knowledge consistency with preliminary information and later introduce a spatiotemporal reasoning layer.
\end{abstract}

Keywords: Formal description, Product-Process, Logic, Ontology, OWL

\section{Introduction}

In complex product development environments, contributions from human can entail mistakes. That is the reason why information along the product lifecycle are currently managed by PLM (Product Lifecycle Management), a computerbased information system. A recurrent issue is that information or knowledge are lacking consistency due to a lack of reasoning capabilities in PLM systems [12]. Relationships between objects have to be simulated to avoid information inconsistency [14]. That is the reason why a theory based on this notion has already been proposed [8]. The theory describes product-process evolution by extracting knowledge from designer's experience. Designers need to be assisted by adavanced knowledge management tools in order to avoid mistakes. Semantic 
web technology has been considered with its language so as to formalize this theory and to represent information in a structured and understandable manner. Ontology is used to capture, represent and reuse knowledge in PLM systems and therefore ensure information and knowledge consistency in product design. The paper is focused on the development of a spatiotemporal ontology in AOD (Assembly-Oriented Design). The objective is to provide a product design description by proactively considering its assembly sequence as early as possible in the product development so as to ensure information and knowledge consistency with preliminary information. The next step will be to add rules to the proposed ontology in order to check information consistency through PLM. Built on this, further efforts will be done so as to introduce DL (Description Logic) and SWRL (Semantic Web Rule Language) rules and then develop a specific reasoning layer.

Firstly, the paper presents, in section 2, a brief literature review on ontology and existing spatiotemporal models. Then, Section 3 describes the proposed formal ontology with OWL (Ontology Web Language) language, which provides product-process associations through semantics and logics. Section 4 introduces a mechanical assembly to illustrate the relevance of the ontology. In section 5 , the advantages and limits of the model are described. Finally, in section 6 , conclusions and future work are given.

\section{Literature Review}

\subsection{Ontology Engineering}

Ontologies address the semantic representation of information for the purpose of storing and exchanging shared knowledge over a worldwide network [6]. An interesting definition is proposed by Gruber [7]: "ontology is an explicit specification of conceptualization". Moreover ontology brings a common vocabulary [11] and a formal aspect enabling information models to be machine interpretable. Inference ontology uses logical deductions on information and ensures the information consistency of the product along the product lifecycle. In mechanical engineering domain, Kim et al. [9] developed an assembly design ontology and an ontology-based assembly design framework in order to facilitate collaborative product development. In this framework, design intent can be well understood among different designers, and applications can reason about assembly knowledge without any semantic ambiguity. Moreover Demoly et al. [2] described product relationships with mereotopological primitives and have implemented it into ontology with OWL-DL and SWRL languages, so as to be machine-interpretable.

\subsection{Ontology Models}

The NIST (National Institute of Standards and Technology) has developed CPM (Core Product Model) to support PLM information [3]. Purely functional reasoning about a product in the conceptual stages of design can be supported by CPM. Then CPM has been extended to cover assembly issues through OAM 
(Open Assembly Model), a standard representation and exchange protocol for assemblies. The NIST interest in knowledge ontologies has led to the transformation of CPM and OAM to an inference model [5]. Then CPM2 has been proposed to support a broad range of information relevant to PLM [4]. Moreover Matsokis et al. have developed an ontology model of the product data and knowledge management Semantic Object Model (SOM) [10] using UML class diagram. SOM is a product item oriented model achieving both an efficient description of the product as it is designed from the manufacturer point of view and a functional structure for storing data of the products lifecycle [12]. So it improves interoperability in PLM.

\section{OWL Implementation of the Spatiotemporal Ontology}

\subsection{Product-Process Knowledge Description in AOD}

A theory, called JANUS (Joined AwareNess and Understanding in assemblyoriented deSign with mereotopology), has already been proposed to describe product-process evolution during the assembly-oriented process with a mereotopologybased theory [8]. The approach formally represents the relationships between regions (Figure 1). Product design is composed of spatial objects (such as parts) linked together by spatial associations and assembly sequence planning is composed of temporal objects (such as assembly operations) linked together by temporal associations. By using spatiotemporal objects (such as swept volumes: space occupied by an object during its move or deformation) and spatiotemporal relationships, spatial objects evolution over time can be understood. Spatial, temporal and spatiotemporal primitives, summed up in Table 1 and Table 2, have been defined in [8] in order to describe possible evolutions during the assembly process. The object evolution depends on its position in space (i.e. give the localization compared to other objects), its position in time (i.e. give the temporal position of the object regarding to others) and its form (i.e. give the object structure). When one attribute (i.e. time, space and form) is modified, a change during the assembly design occurs. Objects and their relationships are considered as entities of information and this information is interconnected to create an ontology [14].

\subsection{Overview of the Research Approach}

The novel approach, called PRONOIA2 (PROduct relatioNships description based On mereotopological theory), is based on the PRONOIA approach [2] and extends it in the temporal and spatiotemporal dimension in order to have a more realistic model. The model aids the designer to check information consistency in the early design phases. Product information can be accessed, stored, served and reused throughout the entire product lifecycle [15] by using a dedicated ontology. The ontology is implemented using Protégé, an ontology management tool [13] and OWL2 (extention of OWL). As shown in Figure 1, the ontology model is divided into three levels, such as the meta-ontology, the domain-ontology and the application-ontology. 
Table 1. Spatial and temporal primitives described in [8]

\begin{tabular}{ll}
\hline Spatial primitive & Temporal primitive \\
\hline Part of $\boldsymbol{P}$ & TemporallyPart of $\boldsymbol{P t}$ \\
InteriorPart of $\boldsymbol{I P}$ & StartTemporallyPart of $\boldsymbol{P t s}$ \\
Crosse $\boldsymbol{X}$ & Finish TemporallyPart of $\boldsymbol{P t f}$ \\
Overlap $\boldsymbol{O}$ & TemporallyInteriorPart of $\boldsymbol{I P t}$ \\
Discrete $\boldsymbol{D}$ & TemporallyPrecede $<$ \\
Tangent $\boldsymbol{T}$ & TemporallyTangent $\boldsymbol{T t}$ \\
Boundary $\boldsymbol{B}$ & TemporallyOverlap $\boldsymbol{O} \boldsymbol{t}$ \\
Straddle $\boldsymbol{S t}$ & TemporallyEqual $=\boldsymbol{t}$ \\
\hline
\end{tabular}

Table 2. Spatiotemporal primitives described in [8]

\begin{tabular}{llll}
\hline \multicolumn{2}{c}{ Spatiotemporal primitives } \\
\hline Kinematic joints & Other evolutions & Technological pairs & \\
\hline Move & SerialPermutation & InterferenceFit & BlindRivet \\
CylindricalOP & ParallelPermutation & GasWelding & ArcWelding \\
PrismaticOP & Addition & Soldering & SpotWelding \\
SphericalOP & Deletion & Brazing & Clinching \\
RevoluteOP & Split & AdhesiveBonding & Crimping \\
PlanarOP & Union & SnapIn & MechanicalFastening- \\
PointContactOP & ChangeOfForm & NailAssembly & ByThreadedFasteners \\
LineContactOP & Deformation & MetalStitching & \\
ScrewOP & Growing & SimpleRivet & \\
Rotation & Decrease & DoubleRivet & \\
\hline
\end{tabular}

\subsection{Meta-ontology Description}

This section introduces the meta-ontology representing objects and their relationships in the three dimensions: spatial, temporal and spatiotemporal. Classes, sub-classes and properties are described to build the knowledge base and illustrated in Figure 2. The model is composed of two top-level types of classes: the physical entity (i.e. region) and the relationship between these entities (i.e. primitive). Spatial and temporal regions and primitives are four different sub-classes of the meta-ontology. As the spatiotemporal dimension depends on the spatial and temporal dimensions, it has been considered as a sub-class of those two dimensions. Classes and sub-classes are linked with properties, which are semantic relationships to facilitate the reuse of existing data, find the inconsistency and errors in data and aids designers to make right decisions by considering complex criteria [1].

\subsection{Assembly-ontology Description}

Based on the meta-ontology, assembly-ontology is defined on Figure 3 in terms of classes, sub-classes and properties in the AOD domain. New introduced classes and sub-classes are represented in bold. The primitive class is not shown as it has already been described in Table 2. This ontology has specific vocabulary linked 


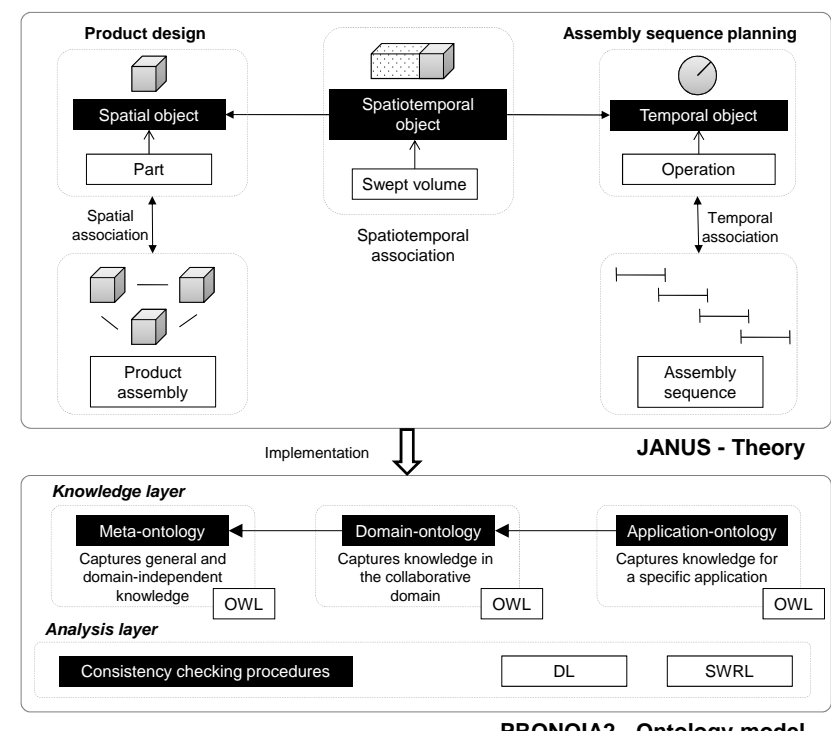

Fig. 1. Research approach of the JANUS theory and of the PRONOIA2 ontology model

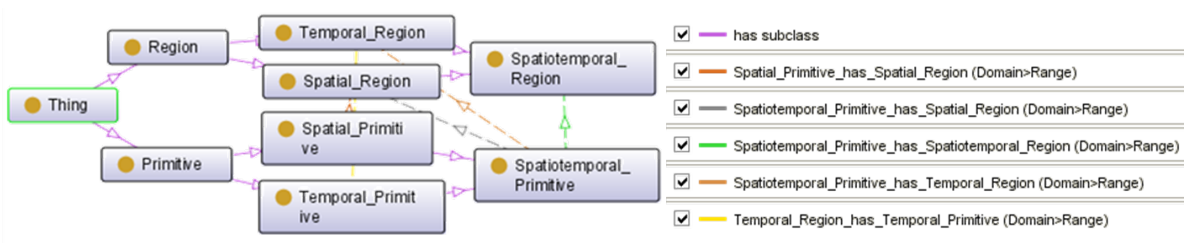

Fig. 2. Classes, sub-classes and properties of the meta-ontology (PRONOIA2)

to the assembly process (such as swept volume), which differentiates it with other domain-ontologies (e.g. manufacturing-ontology). Therefore spatiotemporal primitives, such as kinematic pairs, technological pairs and evolutions inferred during the assembly process, are formalized in this ontology.

\section{Case Study: a Mechanical Assembly}

This section presents a mechanical assembly (see Figure 4) with its related mereotopological descriptions and its related ontology. The case study is composed of six parts. The ASDA algorithm [2] has generated a relevant assembly sequence that is $[1,[3,2,4], 5,6]$. Here the spatial regions 2, 3 and 4 are embedded in a sub-assembly (denoted $S A$ ). Product-process mereotopological description are described in Figure 5 and are based on graph developed in [8]. 


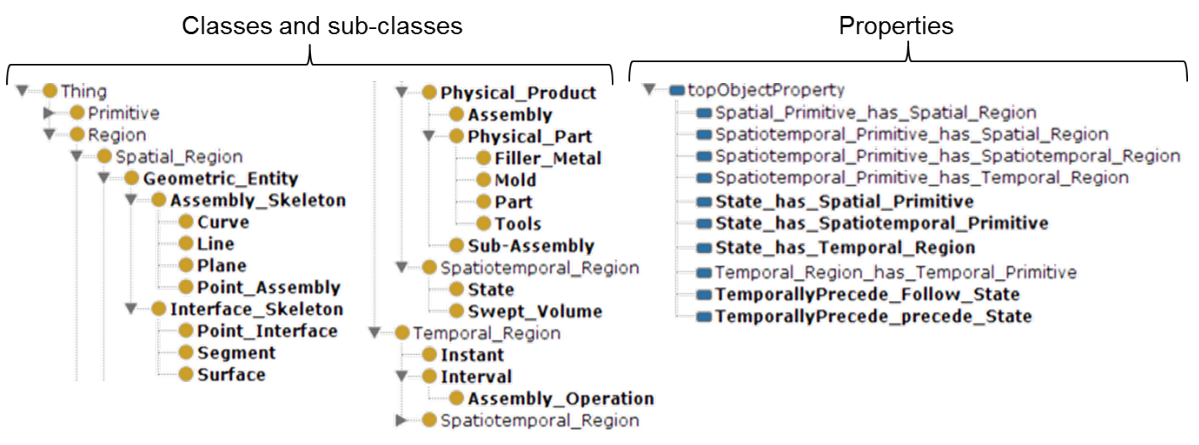

Fig. 3. Classes, sub-classes and properties of the domain-ontology (PRONOIA2)

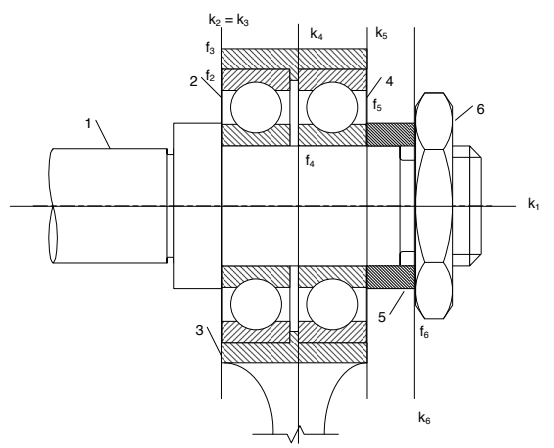

Fig. 4. Cross-section view of the considered mechanical assembly

Spatial regions (i.e. oval), temporal regions (i.e. column) and spatiotemporal regions (i.e. rectangle) are represented and linked with primitives (i.e. line). The third level of the model is created by adding individuals to populate the ontology. A part of the individuals has been shown on Figure 6. The authors have checked that the ontology is composed of all concepts needed in the description of product-process evolution through AOD as well as their consistency.

\section{Discussions}

The proposed ontology is the result of an initial research effort, on which a full ontology covering the whole PLM could be defined. The ontology has been broken down into three levels so that some domain-ontologies (e.g. manufacturingontology...) can be added whenever the authors want. When the manufacturingontology will be added to the ontology, the authors will just have to import the meta-ontology into a new project file. So the proposed ontology has been developed in focusing on the long term. 


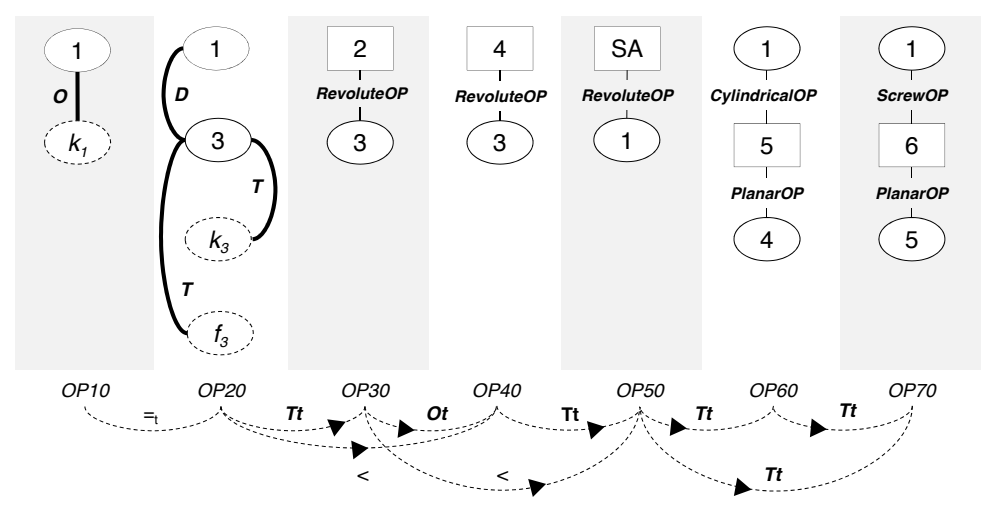

Fig. 5. Understanding product-process integration with semantics and logics

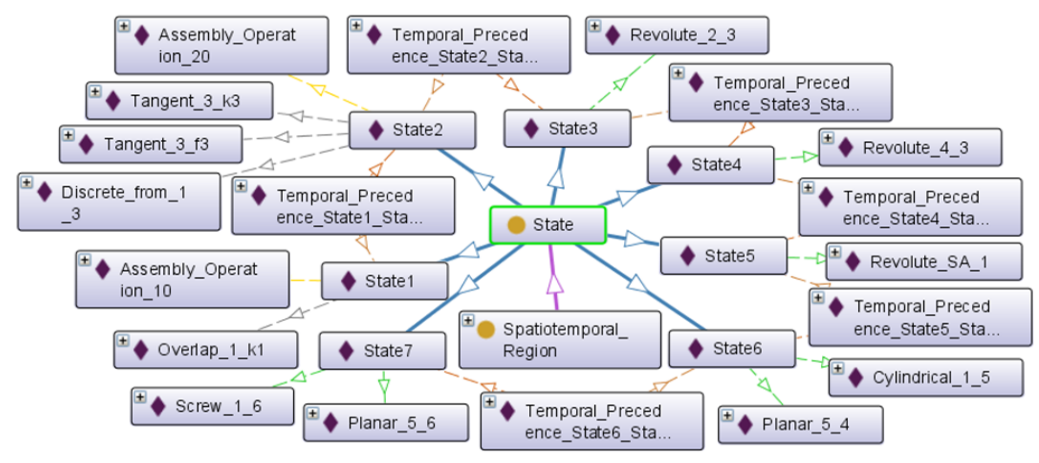

Fig. 6. Application-ontology (PRONOIA2) for the presented case study

\section{Conclusions and Future Work}

Based on a brief state of the art survey on ontology models, this paper has introduced a research effort towards a spatiotemporal ontology in AOD. The proposed PRONOIA2 ontology is based on a mereotopological theory (JANUS), which qualitatively describes product-process definition and evolution. As a consequence, the ontology covers three dimensions, such as spatial, temporal and spatiotemporal, so as to check information consistency issues in AOD. Here, the spatiotemporal dimension has been added in order to be able to describe product-process knowledge and information in a consistent and understandable manner. This dimension aids designers to understand changes during AOD. The ontology model is composed of a meta-ontology, a domain-ontology (in that case the assembly process) and an application-ontology. The JANUS theory is now formalised and machine-interpretable, since an OWL implementation has been done. The actual stake is to get a long term dynamic vision of the space in 
order to facilitate the understanding of assembly and design changes. In future work, rules will be added using SWRL and DL languages so as to reason on spatiotemporal associations within PLM systems (i.e. Product Data Management and Manufacturing Process Management systems). Such efforts will enable the introduction of novel procedures for consistency checking of product-process information and knowledge in PLM.

\section{References}

1. Chang, X.: Ontology Development and Utilization in Product Design. Ph.D. thesis, Virginia Polytechnic Institute and State University (2008)

2. Demoly, F., Matsokis, A., Kiritsis, D.: A mereotopological product relationship description approach for assembly oriented design. Robotics and ComputerIntegrated Manufacturing 28(6), 681-693 (2012)

3. Fenves, S.J., Foufou, S., Bock, C., Sriram, R.D.: CPM: a core model for product data. Journal of computing and information science in engineering 5, 238-246 (2008)

4. Fenves, S., Foufou, S., Bock, C., Sriram, R.: CPM2: a core model for product data. Journal of Computing and Information Science in Engineering 8(1) (2008)

5. Fiorentini, X., Gambino, I., Liand, V., Foufou, S., Rachuri, S., Bock, C., Mani, M.: Towards an ontology for open assembly model. Italie (2007)

6. Fortineau, V., Paviot, T., Lamouri, S.: Improving the interoperability of industrial information systems with description logic-based models - the state of the art. Computers in Industry (2013)

7. Gruber, T.: Ontology. (1993)

8. Gruhier, E., Demoly, F., Gomes, S.: A spatiotemporal mereotopology-based theory for qualitative description in assembly design and sequence planning. J.S. Gero, United Kingdom (2014)

9. Kim, K.Y., Manley, D.G., Yang, H.: Ontology-based assembly design and information sharing for collaborative product development. Computer-Aided Design 38(12), 1233-1250 (2006)

10. Kiritsis, D., Bufardi, A., Xirouchakis, P.: Research issues on product lifecycle management and information tracking using smart embedded systems. Advanced Engineering Informatics 17(3-4), 189-202 (2003)

11. Lee, J., Jeong, Y.: User centric knowledge representations based on ontology for AEC design collaboration. Computer-Aided Design pp. 735-748 (2012)

12. Matsokis, A., Kiritsis, D.: An ontology-based approach for product lifecycle management. Computers in Industry 61(8), 787-797 (2010)

13. Sun, W., Ma, Q.Y., Gao, T.Y., Chen, S.: Knowledge-intensive support for product design with an ontology-based approach. The International Journal of Advanced Manufacturing Technology 48(5-8), 421-434 (2010)

14. Witherell, P., Grosse, I., Krishnamurty, S., Wileden, J.: AIERO an algorithm for identifying engineering relationships in ontologies. Journal Advanced Engineering Informatics pp. 555-565 (2013)

15. Zhong, Y., Qin, Y., Huang, M., Lu, W., Gao, W., Du, Y.: Automatically generating assembly tolerance types with an ontology-based approach. Computer-Aided Design 45(11), 1253-1275 (2013) 